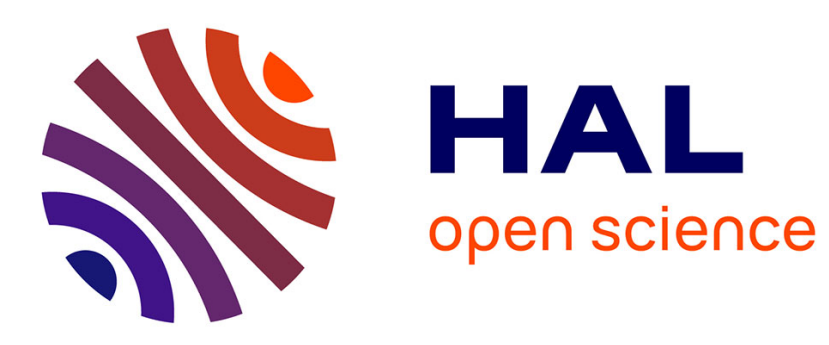

\title{
Le concept d'indice de fibrosité des aliments des ruminants
}

\author{
Daniel Sauvant, J.P. Dulphy, Brigitte Michalet-Doreau
}

\section{To cite this version:}

Daniel Sauvant, J.P. Dulphy, Brigitte Michalet-Doreau. Le concept d'indice de fibrosité des aliments des ruminants. Productions Animales, 1990, 3 (5), pp.309-318. hal-00895914

\section{HAL Id: hal-00895914 https://hal.science/hal-00895914}

Submitted on 1 Jan 1990

HAL is a multi-disciplinary open access archive for the deposit and dissemination of scientific research documents, whether they are published or not. The documents may come from teaching and research institutions in France or abroad, or from public or private research centers.
L'archive ouverte pluridisciplinaire HAL, est destinée au dépôt et à la diffusion de documents scientifiques de niveau recherche, publiés ou non, émanant des établissements d'enseignement et de recherche français ou étrangers, des laboratoires publics ou privés. 
INRA Prod. Anim., 1990, 3 (5), $309-318$

\section{SAUVANT - J.P. DULPHY ${ }^{*}$ Brigitte MICHALET-DOREAU( $\left.{ }^{*}\right)$}

INRA - INA - PG

Station de Nutrition et Alimentation

16, rue Claude-Bernard

75231 Paris Cedex 05

* INRA Station de Nutrition

des Herbivores

Theix 63122 Saint-Genès-Champanelle

\section{Le concept d'indice de fibrosité des aliments
des ruminants des aliments
des ruminants}

La fibrosité de la ration de l'animal ruminant est une caractéristique connue depuis longtemps en raison de ses implications pathologiques et zootechniques. L'évolution de l'élevage, des vaches laitières en particulier, se traduit par une préoccupation de plus en plus fréquente de la fibrosité des rations bien qu'aucune mesure objective et standardisée n'existe pour la caractériser. Dans ce contexte, il a paru utile de faire le point de la connaissance sur le concept de fibrosité et des conséquences pratiques à en tirer.

L'impact des quotas laitiers se traduit, en France plus que dans les autres pays de la CEE, par un accroissement des niveaux individuels de production laitière des vaches, associé à une baisse de leur nombre (CNIEL 1989). Une telle évolution nécessite non seulement d'améliorer la précision des différents systèmes d'unités « hygiéniques » (Journet 1988) aptes à minimiser les risques pathologiques individuels et à mieux pérenniser le capital animal individuel.

L'augmentation des potentiels de production est accompagnée par un apport accru de fourrages jeunes et/ou hachés et d'aliments concentrés et, de ce fait, une baisse de fibrosité de la ration. La fibrosité, classiquement considérée comme un indicateur important de la valeur hygiénique d'une ration (Journet 1988), a une signification concrète; par contre, il est actuellement difficile de l'associer à des caractéristiques facilement mesurables en routine dans des conditions de laboratoire puisqu'elle dépend notamment de la taille des particules

\section{Résumé}

L'indice de fibrosité (IF) des aliments et des rations est égal aux durées d'ingestion et de rumination par $\mathrm{kg}$ de matière sèche $(\mathrm{mn} / \mathrm{kg} \mathrm{MS}$ ). Une baisse de la valeur IF d'un régime se traduit par une moindre motricité ruminale et sécrétion salivaire, elle est en outre associée à une baisse de taux butyreux du lait et à un accroissement des risques de pathologie digestive. Les valeurs IF des aliments s'échelonnent suivant un large éventail ( 0 à $250 \mathrm{mn} / \mathrm{kg} \mathrm{MS}$ ). Dans l'état actuel des connaissances publiées, le concept d'IF peut être utilisé comme indicateur, mais il ne peut pas être considéré comme une unité d'alimentation. alimentaires et de la teneur en parois cellulaires des aliments ingérés. Ce fait explique l'insuffisance des critères tels que la proportion de fourrages ou la teneur en cellulose brute ou en parois cellulaires d'un régime, qui sont parfois utilisés comme critère unique d'estimation de la fibrosité.

\section{1 / Définition et signification physiologique de l'indice de fibrosité}

Devant l'absence de critères analytiques pertinents appliqués aux aliments, des recherches se sont orientées vers la mesure in vivo de paramètres traduisant le travail masticatoire de l'animal.

\section{1 / Définition}

Balch (1971) a proposé d'utiliser la durée de mastication (= ingestion + rumination) pour caractériser la nature et surtout la présentation physique d'un aliment ou d'une ration. Ce concept a été repris par Sudweeks et al (1979, 1981) qui ont combiné, selon différentes proportions, des aliments grossiers et des concentrés de nature variée pour définir une échelle de durées de mastications/kilogramme de matière sèche ingérée (MSI) d'aliment. Cette durée de mastication est généralement appelée Indice de Fibrosité (IF). Le concept d'IF a également été repris par Norgaard (1983) et appliqué dans les tables danoises de valeurs des aliments. Sur la base des valeurs d'Indice de Fibrosité (IFi) d'un ensemble d'aliments i, il est 
théoriquement possible de calculer, par pondération, la valeur de l'IFr du régime (IFr) les regroupant :

$\mathrm{IFr}=\sum \mathrm{pi} . \mathrm{IFi}$

ou MAST $=\sum$ MSIi. IFi

avec $:$ pi $=$ Proportion de MS du régime occupée par l'aliment i $\left(\sum\right.$ pi $\left.=1\right)$
MAST $=$ Durée de mastication $(\mathrm{mn} / \mathrm{j})$

MSIi = Quantité de matière sèche ingérée de l'aliment i $(\mathrm{kg} / \mathrm{i})$.

Cette méthode suppose l'additivité des durées de mastication des différents ingré dients d'un régime. Cette hypothèse est discutée plus loin.

Tableau 1. Caractéristiques d'ingestion et de mastication de rations mixtes par des vaches laitières.

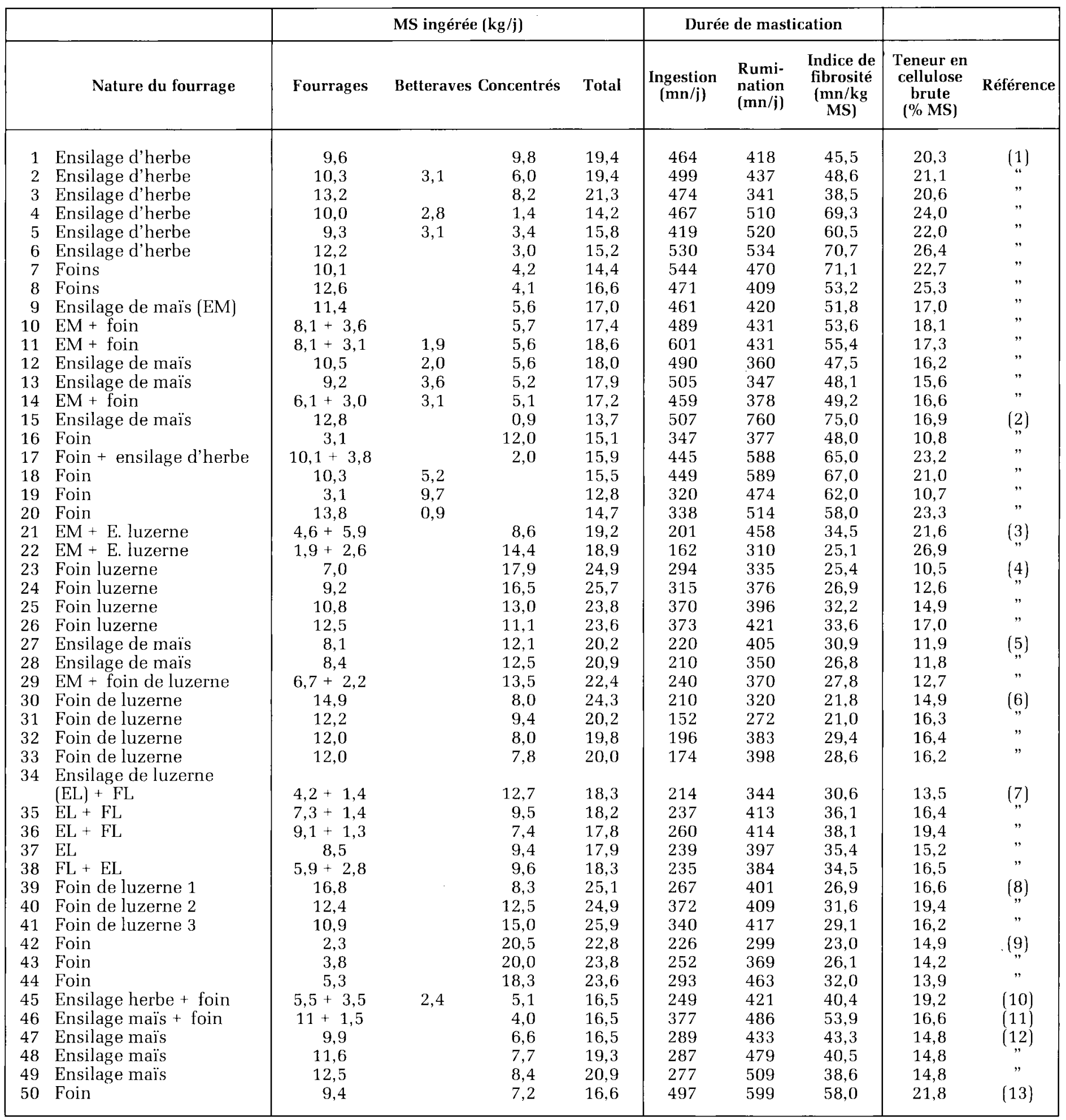

Sources : (1) Dulphy (1989, non publié) ; (2) Remond (1978) ; (3) Grummer et al (1987) ; 4) Woodford et al (1986) ; (5) Woodford Murphy (1988) ; (6) Shaver et al (1986) ; (7) Beauchemin Buchanan-Smith (1989) ; (8) Kaiser et T'omas (1989); (9) Sakurai et al (1989) ; (10) Piatkowski (1982) ; (11) Coulon (1981); (12) Faverdin (1985) : (13) Campling (1966). 
Tableau 2.. Influence de la proportion de paille normale (longue) incluse dans un régime distribué à des vaches sur la durée de mastication et d'autres caractéristiques digestives (Norgaard 1981).

\begin{tabular}{|llrrr|}
\hline \multicolumn{1}{|c}{ Niveau d'incorporation de paille d'orge } & \% MS & $\mathbf{4}$ & $\mathbf{1 0}$ & $\mathbf{2 0}$ \\
\hline Durée de rumination * $^{*}$ & $\mathrm{mn} / \mathrm{j}$ & 130 & 220 & 400 \\
Indice de fibrosité* $^{*}$ & $\mathrm{mn} / \mathrm{kg} \mathrm{MS}$ & 14 & 19 & 32 \\
Contractions réticulo-ruminales* $_{\text {pH ruminal* }}^{*}$ & nbre $/ \mathrm{mn}$ & 1,0 & 1,1 & 1,3 \\
\hline
\end{tabular}

* Les différences sont statistiquement significatives pour ces 4 critères.

Tableau 3. Influence de la taille des particules de la ration sur différents paramètres zootechniques et nutritionnels de la vache pendant les 3 premiers mois de lactation (Woodford et Murphy 1988).

\begin{tabular}{|c|c|c|c|c|c|c|}
\hline $\begin{array}{l}\text { Proportion (MS \%) } \\
\text { de concentré/Haylage/Pellets }\end{array}$ & & $60 / 40 / 12$ & & $60 / 28 / 12$ & & $60 / 12 / 28$ \\
\hline Matière sèche ingérée & $\mathrm{kg} / \mathrm{j}$ & 23,2 & & 23,1 & $>$ & 18,8 \\
\hline Durée de mastication & $\mathrm{mn} / \mathrm{j}$ & 650 & $>$ & 560 & $>$ & 380 \\
\hline Durée de mastication & $\mathrm{mn} / \mathrm{kg} \mathrm{MS}$ & 28,2 & $>$ & 24,1 & $>$ & 20,0 \\
\hline Production de lait brut & $\mathrm{kg} / \mathrm{j}$ & 33,7 & & 35,5 & $>$ & 31,8 \\
\hline Taux butyreux & $\%$ & 30,1 & & 29,3 & $>$ & 25,9 \\
\hline Taux protéique & $\%$ & 31,1 & & 29,9 & & 30,4 \\
\hline Taux de dilution du liquide ruminal & $\% / h$ & 16,6 & $>$ & 13,8 & $>$ & 10,0 \\
\hline Passage de liquide dans le rumen & $1 / \mathrm{j}$ & 243 & $>$ & 193 & $>$ & 137 \\
\hline Taux de passage des particules & $\% / \mathrm{h}$ & 4,0 & & 3,7 & $>$ & 2,7 \\
\hline Rapport acétate/propionate ruminal & & 2,1 & $>$ & 1,8 & & 1,7 \\
\hline pH moyen du rumen & & 5,9 & & 5,7 & & $\mathbf{5 , 6}$ \\
\hline Durée de $\mathrm{pH}\langle 6,0$ & $\mathrm{h}$ & 2,2 & $<$ & 5,2 & $<$ & 7,7 \\
\hline
\end{tabular}

* Les signes $>$ ou $<$ indiquent que les différences sont significatives.

\section{2 / Signification physiologique}

Les incidences pathologiques (acidose, parakératose, fourbure, abcès du foie...) associées à des rations insuffisamment fibreuses ont été fréquemment évoquées depuis plusieurs décennies. Ce n'est que plus récemment que l'IF a été mis en relation avec différentes caractéristiques physiologiques ou zootechniques :

- la durée journalière de mastication (MAST) qui est un paramètre déterminant de l'importance du travail de réduction de la taille des particules. Ainsi Norgaard (1981) a établi une relation entre la MAST (mn/j) et l'indice de fibrosité (IF, mn/kg MS)

MAST $=404+9,12 \mathrm{IF}-0,0245 \mathrm{IF}^{2}$

La non linéarité entre IF et MAST avait déjà été observée par Dulphy et al (1979) sur moutons, elle traduit, au moins en partie l'existence d'une limite maximale de la MAST. Par contre, à partir de mesures effectuées sur des vaches en lactation ingérant des rations mixtes (tableau 1), nous n'avons pas obtenu d'effet quadratique significatif

\section{MAST $=316,3+10,77 \mathrm{IF}$}

$$
(\mathrm{n}=50, \mathrm{R}=0,81, \mathrm{ETR}=79,6 \mathrm{mn})
$$

Ces deux relations indiquent que la durée de mastication s'accroît en moyenne d'environ $10 \mathrm{mn} / \mathrm{j}$ lorsque l'IF augmente de $1 \mathrm{mn} / \mathrm{kg}$ MS.

- la fréquence des contractions ruminales (tableau 2). Norgaard (1981) a calculé une relation moyenne qui associe la motricité ruminale
(MR, contractions/mn) et la durée de mastication (MAST, $\mathrm{mn} / \mathrm{j}$ )

$\mathrm{MR}=0,77+0,001 \mathrm{MAST}$

- la comminution des particules qui conditionne leur passage dans la suite du tube digestif (Ulyatt et al 1984, Baumont 1988).

- le transit des contenus digestifs. Les résultats de Woodford et al (1986) et de Woodford et Murphy (1988, tableau 3) indiquent que le taux de renouvellement des particules et du liquide du réticulo-rumen diminue significativement lorsque les fourrages de la ration sont finement hachés ou remplacés par des pellets. Ces résultats sont à rapprocher de ceux de Poncet et al (1987) montrant que le taux de renouvellement ruminal du foin et du concentré d'une ration est plus lent lorsque la proportion d'aliments concentrés augmente.

- la sécrétion salivaire. Cette sécrétion est continue et présente une valeur basale au cours du repos de 20 à $70 \mathrm{ml} / \mathrm{mn}$. Elle s'intensifie largement pendant l'ingestion (150 à $200 \mathrm{ml} / \mathrm{mn}$ ) et surtout la rumination $(250$ à $300 \mathrm{ml} / \mathrm{mn}$, Erdman 1988). En conséquence, la durée de mastication est le déterminant essentiel des variations des quantités de salive entrant dans le réticulo-rumen. Norgaard (1983) a d'ailleurs proposé une relation entre la sécrétion salivaire (SS, l/j) et la durée journalière de mastication (MAST, mn/j)

\section{$\mathrm{SS}=30+0,20$ MAST}

D'après cette relation, la sécrétion salivaire basale est de $21 \mathrm{ml} / \mathrm{mn}$ et devient égale à 200

\section{La fibrosité d'une ration est associée à un ensemble de caractéristiques physiologiques importantes du ruminant.}


Figure 1. Relation entre la valeur IF d'une ration et le taux butyreux du lait.
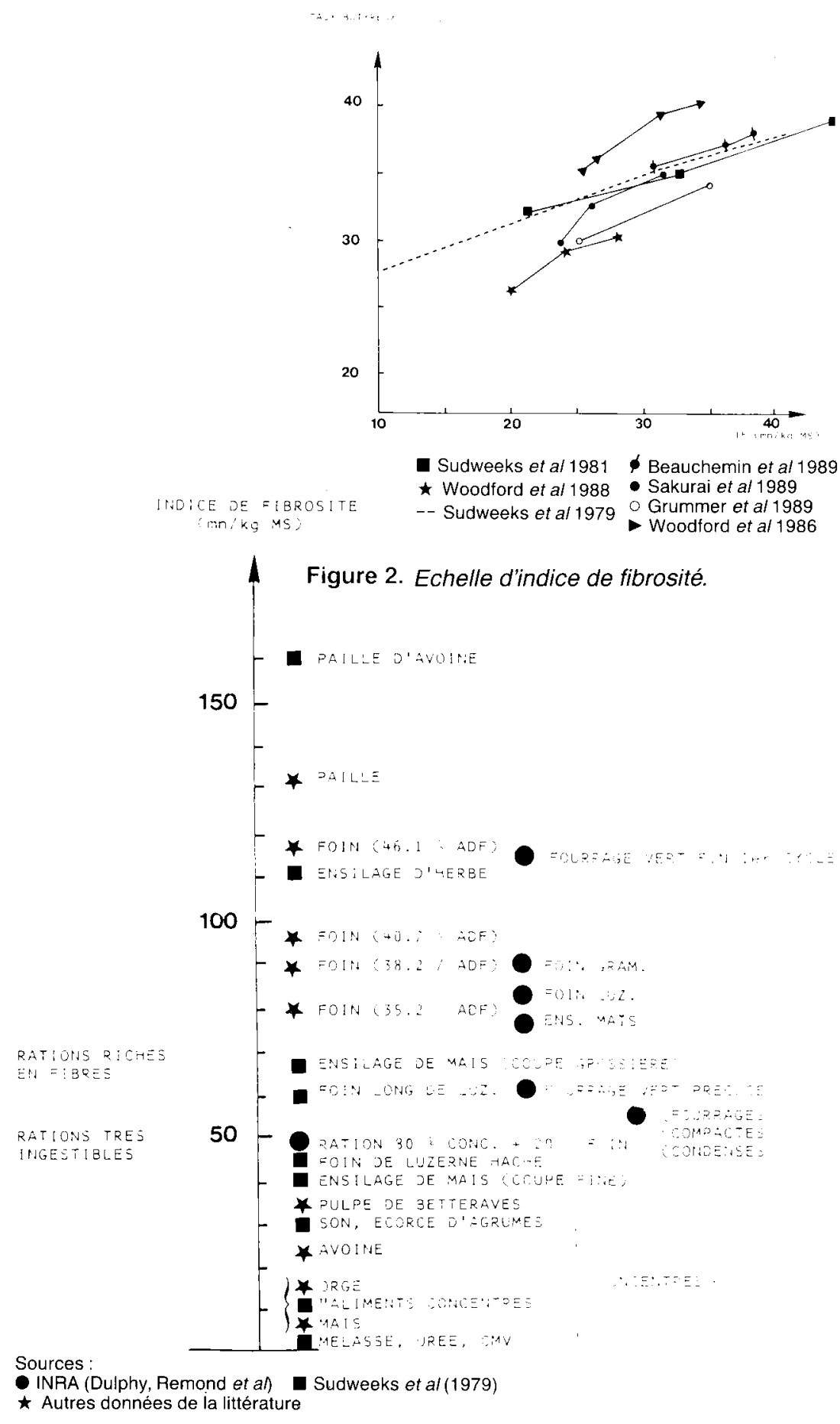

$\mathrm{ml} / \mathrm{mn}$, en période de mastication. Cet apport de salive joue un rôle indispensable dans le bon déroulement de la digestion dans le réticulo-rumen :

- par la fourniture des substances tampons qui tendent à maintenir le $\mathrm{pH}$ à l'intérieur d'une fourchette de valeurs permettant un bon développement des micro-organismes (tableau 3 et Erdman 1988),

- par sa relation avec le renouvellement de la phase liquide du rumen (tableau 3). On considère que celui-ci doit être suffisant $(>8 \% / \mathrm{h})$ pour ne pas handicaper la prolifération microbienne,
- par le recyclage d'urée dont la teneur dans la salive est liée à celle du sang.

- par différents mécanismes intermédiaires digestifs et métaboliques partiellement connus, l'IF de la ration est relié à la valeur du taux butyreux (TB) du lait. La figure 1, établie à partir de données publiées indique que, pour des valeurs d'IFr inférieures à $40 \mathrm{mn} / \mathrm{kg}$ MS, une relation positive systématique associe les valeurs de TB et d'IFr : le TB chute en moyenne de $3 \mathrm{~g} / \mathrm{l}$ pour une baisse d'IF de $10 \mathrm{mn} / \mathrm{kg}$ MS.

\section{2 / Les valeurs d'indice de fibrosité des aliments et des régimes}

\section{1 / Les fourrages}

La figure 2 rapporte les durées de mastication des principaux aliments testés par Sudweeks et al $(1979,1981)$. Cette échelle a été complétée par quelques valeurs obtenues à l'INRA (Rémond 1978) ou citées dans la bibliographie. Les valeurs d'IF des fourrages sont, d'après ces résultats, nettement plus importantes et plus dispersées que celles des aliments concentrés. Par conséquent, la valeur d'IF d'une ration dépend en pratique très largement de sa proportion de fourrage et de la valeur IF de ce dernier.

Il n'y a globalement qu'une faible relation entre les valeurs IF et les teneurs en cellulose brute (CB) des fourrages (figure 3). Cependant, Norgaard (1983) a proposé d'utiliser trois relations moyennes entre les valeurs IF et les teneurs en CB applicables à l'intérieur d'une catégorie de fourrage distinguée par la taille moyenne de ses brins (tableau 4, figure 3 ). Ces relations attribuent $0,75,2,25$ ou $3 \mathrm{mn}$ de mastication par kg de MS et par point p 100 de $\mathrm{CB}$ selon la catégorie de fourrage, elles sont actuellement utilisées dans les tables danoises. La taille des brins n'est cependant pas le seul critère déterminant de la valeur IF des fourrages; ainsi le traitement à la soude de la paille réduit fortement sa durée de mastication par $\mathrm{kg}$ de MS (Teller et al 1990).

\section{2 / Les régimes mixtes}

A partir des données du tableau 1 rapportant des résultats obtenus à l'INRA (Dulphy non publié, Rémond 1978), ou dans la littérature, sur l'ingestion et la mastication de vaches laitières recevant des régimes mixtes ingérés à raison de plus de $12 \mathrm{~kg} \mathrm{MSI} / \mathrm{j}$, on a calculé les paramètres statistiques des différentes caractéristiques d'ingestion, de mastication et de composition des rations rapportées au tableau 5 . Les niveaux de MSI sont compris entre 12,8 et $25,9 \mathrm{~kg} / \mathrm{j}$ et les valeurs de l'IFr entre 21,0 et $75,2 \mathrm{mn} / \mathrm{kg} \mathrm{MSI}$, la teneur en cellulose brute est peu liée à l'IFr du régime ingéré $(\mathrm{R}=+0,50, \mathrm{P}<0,05,1,82 \mathrm{mn}$ par point de $\mathrm{CB} \% \mathrm{MSI}$ ) confirmant ainsi le faible intérêt de ce critère analytique pour évaluer la fibrosité d'un régime. Le tableau 6 des coefficients de corrélation permet de confirmer l'étroitesse des relations entre les critères de durée de mastication première, mérycique ou totale exprimés 


\begin{tabular}{|c|c|c|}
\hline Traitement technologique & $\begin{array}{c}\text { Taille moyenne } \\
\text { des particules }(\mathrm{mm})\end{array}$ & Indice de fibrosité \\
\hline Broyage fin & $<1$ & 4 \\
\hline $\begin{array}{l}\text { Broyage grossier } \\
\text { Céréales a } 7 \text { laties }\end{array}$ & $1<\theta<5$ & 10 \\
\hline Hachage fin & $5<0<10$ & $0,75 \times \mathrm{CB}(1)$ \\
\hline Hachage grossier & $10<\varnothing<50$ & $2,25 \times \mathrm{CB}(1)$ \\
\hline Fourrages longs & $>50$ & $3 \times \mathrm{CB}(1)$ \\
\hline
\end{tabular}

(1) CB : teneur en cellulose brute (\% matière sèche).

par jour ou par kg MSI. Ces différents critères sont négativement et significativement corrélés aux quantités de MSI totale ou de concentrés ingérées, et aux proportions de concentré (PC) ou de concentré + pulpes de betteraves. En fait, il est nécessaire de chercher à dissocier dans les relations les effets spécifiques des niveaux de MSI et de la proportion PC qui sont significativement corrélés $(\mathrm{R}=+0,62, \mathrm{P}<0,05)$. A partir de l'ensemble des données rassemblées, ils ne peuvent pas être dissociés vis-à-vis de leur effet sur la durée quotidienne d'ingestion (DIT, mn/j). Par contre, les durées quotidiennes de rumination (RUT, $\mathrm{mn} / \mathrm{j}$ ).

RUT $=615,6-5,77$ MSI - 187,8 PC

$$
( \pm 3,66) \quad( \pm 58,7)
$$$$
(\mathrm{n}=50, \mathrm{R}=0,64, \mathrm{ETR}=68,7)
$$

et de mastication (MAST, mn / j)

MAST $=1140,9-9,68 \mathrm{MSI}-438,2 \mathrm{PC}$

$$
\text { ( } \mathrm{n}=50, \mathrm{R}=0,66, \mathrm{ETR}=137,5)
$$

sont significativement diminuées et de façon distincte par un accroissement de l'ingestion de MS et de la proportion de concentrés. Il en est de même pour la valeur IFr de la ration :

$\mathrm{IFr}=104,3-2,41 \mathrm{MSI}-0,278 \mathrm{PC}$

$$
( \pm 0,41) \quad( \pm 0,068)
$$$$
(\mathrm{n}=50, \mathrm{R}=0,87, \mathrm{ETR}=7,5 \mathrm{mn})
$$

D'après cette relation, la valeur IFr diminue de $2,41 \mathrm{mn}$ (de 1,59 à $3,23 \mathrm{mn}$ ) lorsque la MSI augmente de $1 \mathrm{~kg}$ et de $2,78 \mathrm{mn}$ (de 1,42 à $4,14 \mathrm{mn}$ ) lorsque la proportion de concentré croît de $10 \%$. Une relation inverse entre le niveau de MSI et la valeur IF d'une même ration a déjà été observée par Coulon (1981) et Faverdin (1985) sur vaches et par Faichney (1984) sur moutons. Dans un même ordre de résultats, Dulphy et al 1979 ont indiqué qu'un accroissement de $1 \mathrm{~kg}$ de la production laitière se traduisait par une baisse moyenne de $1 \mathrm{mn}$ de l'IF du même régime. En réalité, l'influence du niveau de MSI n'est pas linéaire

$\mathrm{IF}=205,43-13,45 \mathrm{MSI}+0,273 \mathrm{MSI}^{2}-0,216 \mathrm{PC}(6)$ $(\mathrm{n}=50, \mathrm{R}=0,90, \mathrm{ETR}=6,82)$ ce qui montre que les valeurs IF d'un même aliment ne sont pas constantes et que les IF ne sont pas des unités additives. D'après cette relation, un accroissement de la MSI entraîne une baisse marginale d'IFr variable selon le niveau de MSI considéré : elle est respectivement de $6,90,5,26,2,53$ et $=0 \mathrm{mn} / \mathrm{kg}$ MS pour 12,15 , 20 et $25 \mathrm{~kg}$ de MSI/j. Cette relation (6) permet en outre de s'attendre à une réduction notable de l'éventail des hiérarchies de valeurs IF des aliments lorsque le niveau de MSI s'accroît.
Figure 3. Relation entre la teneur en cellulose brute $(C B)$ et l'indice de fibrosité des aliments.

Tableau 4. Principes du système danois d'indice de fibrosité (Norgaard 1983).

\section{La teneur en} cellulose brute des aliments ou des rations n'est que médiocrement liée à son indice de fibrosité.

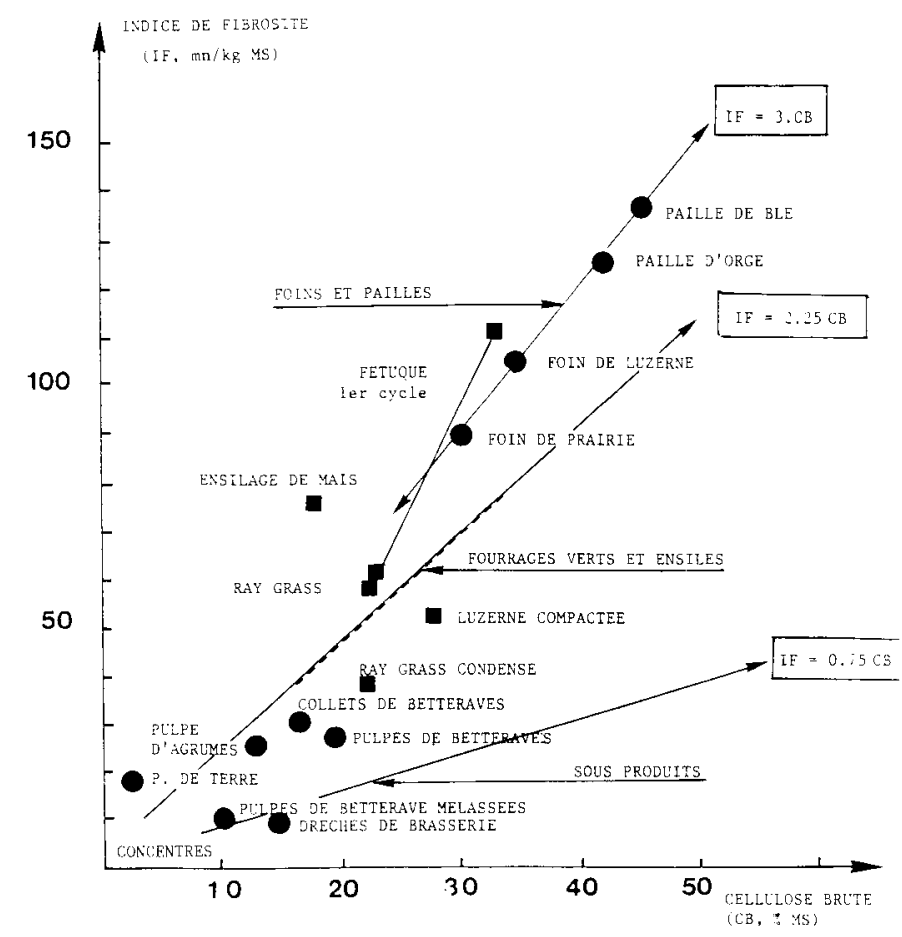

L'influence du niveau de MSI sur l'IF indique que les animaux à haut niveau de production et d'ingestion mastiquent moins leurs rations indépendamment de la proportion de concentré, ce qui va de pair avec des conditions plus favorables à l'acidose latente. D'autre part, étant donné la relation interindividuelle inverse qui associe la durée du transit et la MSI, déterminée par le niveau de production de lait, (Shaver et al 1986 ; Najar et al 1986), il apparaît en conséquence que la sélectivité de l'orifice réticulo omasal (Baumont 1988) doit vraisemblablement être moindre pour les animaux à niveau élevé de production et de MSI. Deswysen et al (1984) ont d'ailleurs observé que la proportion de grosses particules retrouvées dans les fèces augmentait pour un même régime avec le niveau de MSI.

Ces différents résultats montrent qu'il est nécessaire de tenir compte du niveau de MSI pour pouvoir comparer les valeurs d'IF et il paraît dans ce but opportun de se rapporter aux caractéristiques de l'animal standard du système des UE (Dulphy et al 1987). 
Tableau 5. Paramètres statistiques des caractéristiques d'ingestion et de mastication des rations mixtes des vaches laitières (1)

\begin{tabular}{|c|c|c|c|c|c|c|}
\hline & Unité & Sigle & Moyenne & Ecart-type & Min. & Max. \\
\hline Fourrage ingéré & $\mathrm{kg} \mathrm{MSI} / \mathrm{j}$ & FO & 9,93 & 3,06 & 2,3 & 16,8 \\
\hline Betterave ingérée & $"$ & $\mathrm{BE}$ & 0,76 & 1,77 & 0 & 9,7 \\
\hline Concentré ingéré & $"$ & $\mathrm{CO}$ & 8,46 & 5,10 & 0,9 & 20,5 \\
\hline Matière sèche ingérée & $"$ & MSI & 19,14 & 3,51 & 12,8 & 25,9 \\
\hline Durée d'ingestion & $\mathrm{mn} / \mathrm{j}$ & DIT & 346 & 121 & 152 & 601 \\
\hline Durée de rumination & $"$ & RUT & 427 & 89 & 272 & 760 \\
\hline Durée de mastication & $"$ & MAST & 778 & 183 & 424 & 1267 \\
\hline Indice d'ingestion & $\mathrm{mn} / \mathrm{kg} \mathrm{MSI}$ & IIN & 19,4 & 8,9 & 7,5 & 37,8 \\
\hline Indice de rumination & $"$ & IRU & 23,8 & 8,8 & 13,1 & 55,4 \\
\hline Indice de fibrosité & $"$ & IF & 42,4 & 15,6 & 21,0 & 75,2 \\
\hline Teneur en cellulose brute & $\% \mathrm{MS}$ & $\mathrm{CB}$ & 17,5 & 4,1 & 10,5 & 26,9 \\
\hline Cellulose brute ingérée & $\mathrm{kg} / \mathrm{j}$ & CBI & 3,26 & 0,71 & 1,36 & 5,08 \\
\hline \% concentré & $\%$ & $\mathrm{PC}$ & 41,7 & 21,5 & 5,0 & 90,0 \\
\hline$\%$ betteraves + concentré & $\%$ & PBC & 46,6 & 18,6 & 5,0 & 90,0 \\
\hline
\end{tabular}

(1) A partir des données du tableau 1.

Les données expérimentales du tableau 1 permettent d'autre part d'estimer à l'aide du 'modèle d'ajustement (2) les valeurs IF moyennes des aliments utilisés au sein de ces rations mixtes. Le tableau 7 regroupe les principaux résultats obtenus, il en ressort un « tassement » notable des échelles de valeur IF des aliments par rapport à la figure 2 : les foins de pré et l'ensilage d'herbe se situent, à un extrême, vers $65 \mathrm{mn} / \mathrm{kg}$ MSI et le concentré, à l'opposé, vers $32 \mathrm{mn} / \mathrm{kg}$ MSI. Ce tassement provient en particulier de l'influence négative et non linéaire du niveau de MSI sur l'IF. Les graminées présentent en moyenne des valeurs IF supérieures de plus de $20 \mathrm{mn}$ à la luzerne, l'ensilage de maïs occupant une position intermédiaire entre les deux. Un effet significatif, lié à la source expérimentale et/ou au type de four- rage ou de concentré, apparaît dans la variation résiduelle de l'équation de prévision des valeurs IF. Cet effet peut être également causé par des différences dans la méthode de mesure du comportement alimentaire des animaux.

Le même modèle d'ajustement appliqué aux indices d'ingestion (IIN) et de rumination (IRU) indique que ces derniers sont globalement mieux expliquées par les aliments ingérés (tableau 7). Par contre, mis à part le concentré, les variations des durées d'ingestion sont plus importantes que celles de la rumination. Les données du tableau 7 permettent également de constater que les différences de valeurs IF moyennes des aliments considérés sont plus liées à celles des indices d'ingestion que de rumination.

Matrice des corrélations entre les caractères du tableau 1 (1).

\begin{tabular}{|c|c|c|c|c|c|c|c|c|c|c|c|c|c|c|c|}
\hline \multicolumn{16}{|c|}{ CORRÉLATIONS } \\
\hline & FO & $\mathrm{BE}$ & $\mathrm{CO}$ & MSI & DIT & RUT & IF & CB & $\mathrm{BC}$ & MAST & IIN & IRU & CBI & PBC & PC \\
\hline FO & 1.000 & & & & & & & & & & & & & & \\
\hline $\mathrm{BE}$ & $-0,242$ & 1,000 & & & & & & & & & & & & & \\
\hline $\mathrm{CO}$ & $-\underline{0,487}$ & $\underline{0,484}$ & 1,000 & & & & & & & & & & & & \\
\hline MSI & 0,043 & - $\underline{0,419}$ & $\underline{0,797}$ & 1,000 & & & & & & & & & & & \\
\hline DIT & 0,262 & 0,262 & $-\underline{0,517}$ & $-\underline{0,400}$ & 1,000 & & & & & & & & & & \\
\hline RUT & $\underline{0,288}$ & 0,177 & $-\underline{0,595}$ & $-\underline{0,529}$ & $\underline{0,479}$ & 1,000 & & & & & & & & & \\
\hline IF & 0,180 & $\underline{0,421}$ & $-\underline{0,808}$ & $-\underline{0,817}$ & $\underline{0,784}$ & $\underline{0,767}$ & 1,000 & & & & & & & & \\
\hline CB & $\underline{0,360}$ & $-0,013$ & $-\underline{0,485}$ & $-\underline{0,403}$ & $\underline{0,418}$ & $\underline{0,361}$ & $\underline{0,498}$ & 1,000 & & & & & & & \\
\hline BC & $-\underline{0,645}$ & $-0,154$ & $\underline{0,939}$ & $\underline{0,736}$ & - $\underline{0,481}$ & $-\underline{0,602}$ & $-\underline{0,747}$ & $\underline{0,553}$ & 1,000 & & & & & & \\
\hline MAST & $\underline{0,316}$ & 0,262 & $-\underline{0,636}$ & $-\underline{0,526}$ & $\underline{0,905}$ & $\underline{0,808}$ & $\underline{0,899}$ & $\underline{0,456}$ & $-\underline{0,615}$ & 1,000 & & & & & \\
\hline IIN & 0,188 & $\underline{0,364}$ & $-\underline{0,706}$ & - $\underline{0,691}$ & $\underline{0,927}$ & $\underline{0,617}$ & $\underline{0,945}$ & $\underline{0,474}$ & $-\underline{0,654}$ & $\underline{0,923}$ & 1,000 & & & & \\
\hline IRU & 0,152 & $\underline{0,343}$ & $\underline{0,756}$ & - $\underline{0,803}$ & $\underline{0,501}$ & $\underline{0,913}$ & $\underline{0,894}$ & $\underline{0,398}$ & $-\underline{0,719}$ & $\underline{0,779}$ & $\underline{0,738}$ & 1,000 & & & \\
\hline CBI & $\underline{0,390}$ & - $\underline{0,304}$ & 0,119 & $\underline{0,366}$ & 0,097 & $-0,076$ & $-0,156$ & 0,689 & 0,016 & 0,028 & $-0,079$ & $-0,244$ & 1,000 & & \\
\hline PBC & - $\underline{0,844}$ & 0,063 & $\underline{0,796}$ & $\underline{0,458}$ & $-\underline{0,463}$ & $-\underline{0,587}$ & $-\underline{0,605}$ & $\underline{0.558}$ & $\underline{0,923}$ & $-\underline{0.596}$ & $-\underline{0,546}$ & $-\underline{0,589}$ & $-0,179$ & 1,000 & \\
\hline PC & $-\underline{0,575}$ & - $\underline{0,523}$ & $\underline{0,960}$ & $\underline{0,640}$ & $-\underline{0,522}$ & $-\underline{0,614}$ & $-\underline{0,760}$ & $\underline{0,456}$ & $\underline{0,879}$ & - $\underline{0,649}$ & $-\underline{0,664}$ & - $\underline{0,716}$ & $\underline{0,039}$ & $\underline{0,813}$ & 1,000 \\
\hline
\end{tabular}


Tableau 7. Calcul des valeurs IF moyennes des aliments inclus dans des rations mixtes de vaches laitières (1).

\begin{tabular}{|c|c|c|c|c|c|c|c|}
\hline \multirow[t]{2}{*}{ Aliment } & \multirow{2}{*}{$\begin{array}{l}\begin{array}{l}\text { Niveau } \\
\text { de MSI }\end{array} \\
(\mathrm{kg} / \mathrm{j})\end{array}$} & \multicolumn{2}{|c|}{ Durées de mastication } & \multicolumn{2}{|c|}{ Durées d'ingestion } & \multicolumn{2}{|c|}{ Durées de rumination } \\
\hline & & $a^{(2)}$ & IF (a) & $a^{121}$ & IIN $^{1: 31}$ & $a^{(2)}$ & $\operatorname{IRU}^{(3)}$ \\
\hline Betteraves & $0,76(1,79)$ & $2,76 \quad(17,3)$ & 45,3 & $9,72(12,74)$ & 30,1 & $-6,95 \quad(9,92)$ & 18,1 \\
\hline Concentré & $8,46(5,14)$ & $-9,80 \quad(8,15)$ & 32,7 & $-0,93 \quad(5,99)$ & 19,4 & $-8,87 \quad(4,67)$ & 16,2 \\
\hline Foin graminées & $1,97(3,69)$ & $23,43(14,42)$ & 65,9 & $18,48 \quad(10,6)$ & 38,8 & $4,95 \quad(8,26)$ & 30,0 \\
\hline Foin de luzerne & $2,80(5,05)$ & $2,75 \quad(9,74)$ & 45,3 & $4,59(7,17)$ & 24,9 & $-1,84 \quad(5,58)$ & 23,3 \\
\hline Ensilage d'herbe & $1,48(3,62)$ & $22,11(13,21)$ & 64,6 & $20,57 \quad(9,71)$ & 40,9 & $1,55 \quad(7,57)$ & 26,6 \\
\hline Ensilage de luzerne & $0,87(2,32)$ & $-2,9(16,83)$ & 40,4 & $-0,41(12,37)$ & 19,9 & $-1,67 \quad(9,64)$ & 23,4 \\
\hline Ensilage de maïs & $2,81(4,46)$ & $16.31(12,92)$ & 58,8 & $14,37 \quad(9,5)$ & 34,7 & $1,94 \quad(7,40)$ & 27,0 \\
\hline Constante (mn/j) & & 772,9 & & 346,1 & & 426,7 & \\
\hline Corrélation multiple & & 0,77 & & 0,72 & & 0,66 & \\
\hline $\mathrm{ETR}(\mathrm{mn} / \mathrm{j})$ & & 124,2 & & 91,0 & & 71,0 & \\
\hline
\end{tabular}

"' Les valeurs entre parenthèses sont des écarts-types. Les régressions sont calculées avec les variables explicatives centrées à partir des données du tableau 1.

${ }^{22}$ Coxfficients de régression.

${ }^{1: 11}$ Indices IF, IIN et IRU (tableau 5] calculés en mn/kg MSI pour un niveau de MSI de $17 \mathrm{~kg} / \mathrm{j}$.

Tableau 8. Valeurs d'indice de fibrosité proposées par Sudweeks et al (1979) pour les aliments concentrés.

\begin{tabular}{|lc|}
\hline & IF (mn/kg MS) \\
\hline Luzerne déshydratée condensée & 37 \\
Pulpes d'agrumes & 31 \\
Enveloppes de graine de coton & 30 \\
Enveloppes de riz broyées & 16 \\
Epis de maïs en pellets & 15 \\
Orge broyée & 15 \\
Avoine broyée & 12 \\
Aliment composé en pellets & 12 \\
Sorgho broyé & 11 \\
Blé broyé & 10 \\
Remoulages & 8 \\
Coques de soja & 8 \\
Tourteaux broyés & 6 \\
Maïs grain broyé & 5 \\
Mélasse, urée & 0 \\
\hline
\end{tabular}

\section{3 / Les aliments concentrés}

A notre connaissance, aucune étude n'a cherché, à ce jour, à reprendre l'approche systématique de Sudweeks et al (1981) pour déterminer les valeurs d'IF des aliments concentrés les plus utilisés dans l'alimentation des ruminants. En outre, les données publiées de façon éparse sur la question laissent apparaître des contradictions susceptibles d'aboutir à des différences non négligeables de valeur d'Indice de Fibrosité d'un régime riche en concentrés. Une grande prudence doit donc être appliquée dans la manipulation de ces valeurs chiffrées.

Des preuves expérimentales des différences d'IF entre concentrés existent. Ainsi dans un travail réalisé sur moulons, Guérin et Dulphy (1984) ont calculé les durées de mastication de régimes ayant des niveaux d'apport variables de maïs grain et de pulpes de betteraves. La reprise par régression multiple des résultats obtenus avec 10 régimes en combinant des niveaux variables de foin et de ces deux ali- ments a permis de calculer que la durée quotidienne de mastication s'accrôit de :

$18,1 \mathrm{mn} / 24 \mathrm{~h}$ par $\mathrm{g}$ de MSI de foin $/ \mathrm{kg} \mathrm{p}^{\mathrm{n}, \bar{\pi} \mathrm{s}}$ $4,3 \mathrm{mn} / 24 \mathrm{~h}$ par g de MSI de maïs $/ \mathrm{kg} \mathrm{p}^{n, 7, \pi}$ 2,6 mn $/ 24 \mathrm{~h}$ par g de MSI de pulpes $/ \mathrm{kg}^{\mathrm{g}} \mathrm{p}^{1.55}$

Le maïs entraînerait donc chez le mouton une durée de mastication légèrement plus importante que la pulpe de betterave en relation possible avec des phénomènes d'interaction digestive plus marqués pour le maïs.

Le tableau 8 rapporte l'éventail des valeurs d'IF proposées par Sudweeks et al (1981) pour les aliments concentrés: de 0 à $37 \mathrm{mn} / \mathrm{kg}$ de MSI. Les tables danoises sont moins nuancées puisqu'elles attribuent une valeur d'IF de $4 \mathrm{mn}$ / kg MS à tous les aliments concentrés excepté les valeurs de $10 \mathrm{mn} / \mathrm{kg}$ MS pour les drêches de brasserie, $25 \mathrm{mn} / \mathrm{kg}$ MS pour les pulpes , d'agrumes et $30 \mathrm{mn} / \mathrm{kg}$ MS pour les pulpes de betteraves déshydratées. Ces dernières valeurs sont proches de celles que l'on obtient pour le concentré (32,7 mn, tableau 7) par l'interprétation des données du tableau 1. Rappelons que l'équation (6) indique que cette valeur IF moyenne du concentré varie selon le niveau de MSI. Elle est ainsi de 43,24 et $18 \mathrm{mn} / \mathrm{kg}$ MSI pour des niveaux respectifs de MSI de 15, 20 et $25 \mathrm{~kg} / \mathrm{j}$.

Les valeurs IF des différents concentrés publiées ont été reliées à la teneur probable en parois cellulaires (INRA 1988) de ces aliments (figure 4). La disposition des points dans cette figure 4 indique qu'il n'est pas possible de prévoir actuellement précisément les valeurs d'IF des aliments concentrés issus d'un traitement physique comparable à l'aide d'un critère lié aux constituants pariétaux. Néanmoins, il semble que les aliments concentrés riches en parois cellulaires présentent des valeurs d'IF plus importantes que les autres, le coefficient de proportionnalité étant de l'ordre de $5 \mathrm{mn}$ d'IF pour $10 \%$ de parois cellulaires. A propos de la prévision des valeurs d'IF des concentrés, il est 
Figure 4. Relation entre l'indice de fibrosité et la teneur en parois cellulaires de quelques aliments concentrés.

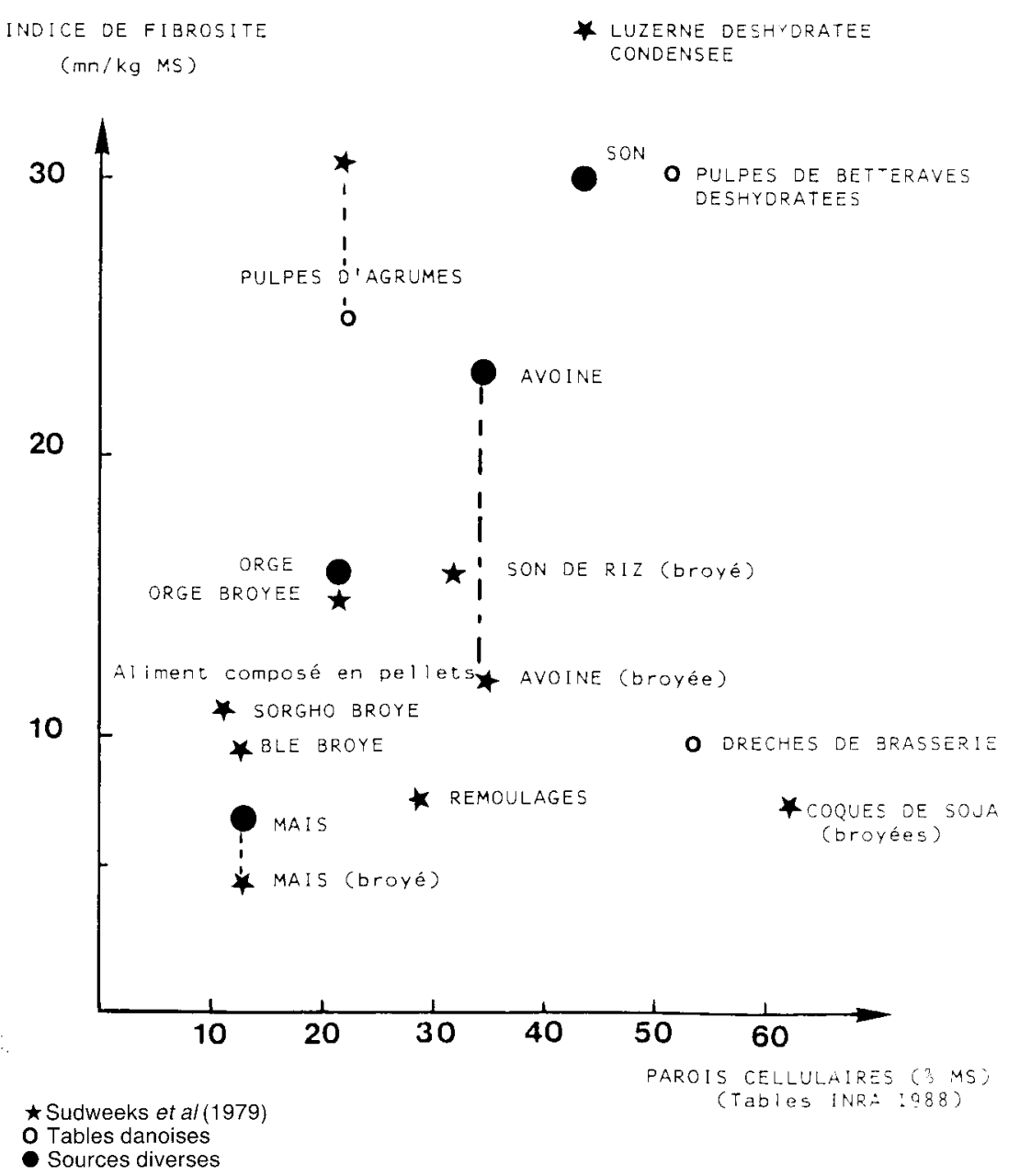

maximale de la proportion de concentré à ne pas dépasser dans le régime.

D'autre part, aucune recommandation de ce type n’a encore été faite à notre connaissance pour des ovins et des caprins sachant que, pour une même ration, les petits ruminants ne présentent pas les mêmes durées de mastication que les bovins (Embrecht et Deswysen 1990 ; Dulphy et al 1990). Enfin, pour une même ration et un même type d'animal, il convient de signaler l'existence de variations individuelles marquées (Faverdin 1985) qui sont, en partie au moins, déterminées génétiquement (Amouche et Deswysen 1990). Ces différents aspects, ainsi que d'autres facteurs susceptibles d'interférer avec le concept d'IF, ont été récemment développés par Deboever et al (1990).

\section{4 / Quels inconvénients a une durée de mastication excessive?}

Il est assez difficile de cerner des inconvénients spécifiques d'une durée excessive de mastication. La seule approche réalisée à ce propos nous paraît être le travail du groupe de Cornell. Van Soest et al (1984) ont en effet cherché à appliquer des coefficients de réduction de la valeur énergétique (" discount factors») individualisés pour chaque matière première pour tenir compte, d'après ces auteurs, de la dépression de la valeur alimentaire avec l'augmentation du niveau d'ingestion liée au travail de mastication par son rôle :

- d'accroissement de l'extra-chaleur,

- d'accélération du transit permis par une réduction plus efficace de la taille des particules.

L'équation de prédiction proposée pour obtenir le coefficient de réduction (CR) est :

$\mathrm{CR}=0,033+0,132 \mathrm{NDF}-0,033 \mathrm{TDNm}$ avec NDF : la teneur en parois cellulaires (en \% de la MS)

probable que des technirues telles que la mesure du rendement d'un broyeur ou de l'énergie nécessaire au broyage, déjà proposées il y a plus de 20 ans pour les fourrages (Chenost 1966), pourraient fournir des informations simples, mais très utiles.

\section{3 / Quelle recommandation minimale d'IF ?}

Les données actuellement disponibles ne permettent pas de mettre au point un système précis d'indice de fibrosité.
La valeur ou le besoin minimal d'IF à retenir pour un type d'animal donné demandent à être précisés. Pour la vache laitière, la valeur de $30 \mathrm{mn} / \mathrm{kg}$ MS a été proposée par les chercheurs danois (Norgaard 1986) sans toutefois préciser s'il s'agissait d'une limite stricte ou d'un ordre de grandeur. La figure 1 plaide pour ce dernier point de vue car le TB ne révèle pas de " saut de variation » lorsque la valeur d'IF d'une ration est inférieure à $40 \mathrm{mn} / \mathrm{kg} \mathrm{MS}$. En outre, il serait nécessaire de préciser la relation entre la valeur IF du régime et ses risques d'incidence pathologiques. Si une valeur minimale à recommander d'IFr était admise, les relations (5) ou (6) permettraient de connaître, pour différents niveaux d'ingestion de MSI, la valeur
TDNm : la valeur TDN, en \%, mesurée à l'entretien.

Ce coefficient de réduction s'applique à la valeur TDN de l'aliment en fonction du niveau de production (NP)

TDNnp = TDNm - (NP - 1) $(\mathrm{CR} \times \mathrm{TDNm} / 100)$

Cette équation accorde un poids dépressif important à la paroi cellulaire. L'idée d'appliquer des coefficients correcteurs individualisés pour les aliments paraît être intéressante ; cependant, les mécanismes évoqués et les valeurs proposées de CR demandent à être expérimentalement démontrés.

\section{5 / La relation entre les valeurs UE et IF des aliments}

Il est logique de penser qu'une relation positive associe les valeurs d'encombrement (UE) et les indices de fibrosité des fourrages. La figure 5 , établie pour quelques aliments à partir des tables danoises et INRA 1988, confirme le sens 
de cette relation, mais permet également d'apprécier son manque de précision; en outre, il apparaît que, pour l'ensemble des fourrages verts classiques, la variation d'IF par UEL serait bien plus importante que ne le laisse penser la relation générale ( $=450 \mathrm{mn} / \mathrm{UEL}$ vs $=$ $150 \mathrm{mn}$ / UEL). La relation moyenne approximative

$\mathrm{IF}=150$ UEL -75

permet de passer d'une échelle de valeurs à l'autre à condition d'attribuer une valeur fixe aux aliments concentrés $(0,50<\mathrm{UEL}<0,70)$. Elle permet, par exemple, de vérifier que la recommandation IF $>30 \mathrm{mn} / \mathrm{kg}$ MS, citée plus haut, correspond à une valeur UEL/kg MS de la ration $>0,70$, limite proposée par Journet (1988).

\section{Conclusion}

L'indice de fibrosité semble être, dans l'état actuel des connaissances, le meilleur critère synthétique pour évaluer l'importance du travail masticatoire d'un ruminant et des régulations physiologiques digestives qui lui sont associées. Plusieurs essais ont démontré l'influence prépondérante des fourrages sur le travail de mastication nécessité par une ration et les quelques mesures spécifiques d'Indice de Fibrosité effectuées jusqu'alors permettent d'avoir un ordre d'idée de l'éventail des valeurs IF des principaux aliments. On dispose de résultats de mesures de durées de mastication de vaches laitières placées dans des conditions proches de la pratique. Les animaux à haut niveau de production et d'ingestion investiraient moins de temps à mastiquer leur ration, cette tendance est particulièrement marquée quand cette durée est exprimée par kg de MSI. En conséquence, il apparaît utile de ne pas choisir les aliments de ces animaux que sur la base de leur concentration en UFL, PDI et UE, mais de chercher à prendre également en compte leur valeur IF et peut-être également d'autres critères tels que leur pouvoir acidogène intrinsèque (Malestein et al 1984). Les données de la littérature permettent également de démontrer l'influence de l'IFr sur le TB du lait.

Un autre acquis important est la démonstration du fait que la teneur en cellulose brute du régime ne constitue pas un critère satisfaisant de prédiction de la durée de mastication des aliments et des rations. Les études les plus récentes publiées outre Atlantique ont d'ailleurs délaissé ce critère au profit de la teneur en paroi cellulaire, appréciée par le résidu NDF, de la ration. Cependant, les résultats obtenus avec ce paramètre ne sont pas encore suffisamment nombreux et concluants pour être intégrables au sein de recommandations.

Un objectif logique serait de mettre au point un système d'IF, l'état actuel des connaissances et l'interprétation des données publiées sur vaches laitières permettent de définir les principales questions à aborder dans ce but :

- la définition de la capacité de mastication des animaux et de ses seuils minimum et maximum,
Figure 5. Relations entre l'indice de fibrosité et la valeur d'encombrement (UEL).

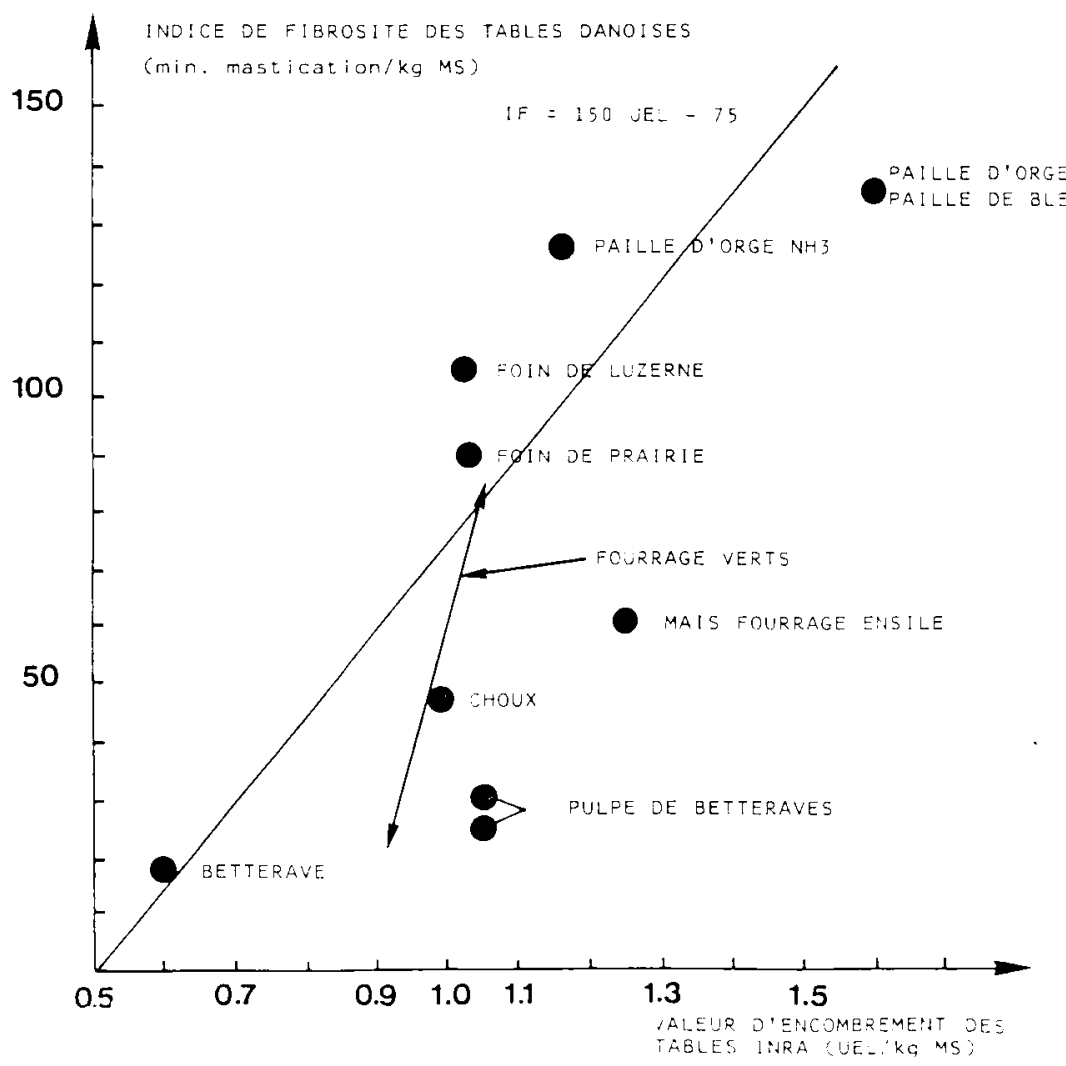

- la définition d'un protocole standard de mesure systématique des IF, basé sur l'utilisation d'un animal de référence en production (VLS à $25 \mathrm{~kg}$ de lait/jour?),

- la détermination des lois de corrections à appliquer pour interpréter les données obtenues avec des animaux ayant des caractéristiques différentes du standard,

- l'analyse des phénomènes d'interactions entre les aliments d'une même ration. Cet aspect et le précédent concernent le manque d'additivité des valeurs IF des aliments,

- la prévision à l'aide de méthodes simples et peu coûteuses des valeurs IF des aliments.

\section{Références bibliographiques}

AMOUCHE E., DESWYSEN AG, 1990. Influence du mode de logement et de l'ascendance paternelle sur le comportement alimentaire mérycique et la distribution de la taille des particules fécales chez les ovins. Reprod. Nutr. Develop. (sous presse).

BALCH C.C., 1971. Proposal to use time spent chewing as an index of the extent to which diets for ruminants process the physical property of fibrousness characteristic of roughages, Br. J. Nutr., 26, 383-392.

BEAUCHEMIN K.A., BUCHANAN-SMITH J.G., 1989 Effect of dietary neutral detergent fiber concentration and supplementary long hay on chewing activities and milk production of dairy cows. J. Dairy Sci., 72, 2288-2300.

BAUMONT R., 1988. Ftat de réplétion du réticulo-rumen et ingestion des fourrages, Thèse de Doctorat de l'INAPG. CHENOST M., 1966. L'indice de fibrosité des foins: mesures et relations avec la valeur alimentaire, Ann. Zootech., 15, 253-257. 
COULON J.B., 1981. Capacité d'ingestion des vaches laitières en début de lactation, thèse de Docteur Ingénieur, ENSA Montpellier.

C.N.I.E.L., 1989. L'économie Iaitière en chiffres, Ed. C.N.I.E.L. Paris

DE BOEVER J.L., ANDRIES J.I., DE BRABANDER D.L. COTTYN B.G., BUYSSE E.X., 1990. Chewing activity of ruminants as a measure of physical structure. A review of factors affecting it. Anim. Feed Sci. Technol., 27, 281-291. DESWYSEN A.G., BRYER D.C., VANBELLE M., 1984. Circadian rumination quality and voluntary silage intake in sheep and cattle. Can. J. Ánim. Sci. Suppl., 64, 341-342.

DULPHY J.P., REMOND B., THERIEZ M., 1979. Ingestive behaviour and related activities in ruminants. In « Digestive physiology and metabolism in ruminants " Ed. Ruckebusch et Thivend, MTP Press, p 103-122.

DULPHY J.P., FAVERDIN P., MICOL D., BOCQUIER F. 1987. Révision du système des unités d'encombremen (UE), Bull. Tech. CRZV Theix, INRA, 70, 35-48.

DULPHY J.P., CARLE B., DEMARQUILLY C., 1990. Quantités ingérées et activités alimentaires comparées des ovins, bovins et caprins recevant des fourrages conservés avec et sans aliment concentré. I - Etude descriptive, Ann. Zootech. (soumis)

EMBRECHTS J.M., DESWYSEN A.G., 1990. Influence de la teneur en composants pariétaux (NDF) des ensilage d'herbe sur le comportement alimentaire et mérycique des ovins et des bovins. Reprod. Nutr. Develop. (sous presse)

ERDMAN R.A., 1988. Dietary buffering requirements of the lactating dairy cow: a review, J. Dairy Sci., 71, 32463266 .

FAICHNEY G.J, 1984. The kinetics of particulate matter in the rumen, , in "Control of digestion and metabolism in ruminants", Ed. Milligan, Grovum, Dobson, PrinticeHall, p 173-195.

FAVERDIN P., 1985. Regulation de l'ingestion des vaches laitières en début de lactation : étude du rôle de l'insuline. Thèse Doct. Ing. INAPG, 131 p.

GRUMMER R.R., JACOB A.L., WOODFORD J.A., 1987. Factors associated with variation in milk fat depression resulting from high grains diets fed to dairy cows. I. Dairy Sci., 70, 613-619.

GUERIN H., DULPHY G.I... 1984. Influence de l'apport complémentaire de mais, de pulpe de betteraves ou de mélasse sur la valeur alimentaire d'un foin. Ann. Zootech., 33, 509-532.

INRA, 1988. Alimentation des bovins ovins et caprins, Ed. R. Jarrige, INRA Paris, $471 \mathrm{p}$.

JOURNET M., 1988. Optimisation des rations. in "Ali mentation des bovins, ovins et caprins". Ed. R. Jarrige INRA Paris, $p$ 121-133.

KAISER R.M., COMBS D.K., 1989. Utilization of three maturities of alfalfa hay by dairy cows fed rations tha contain similar concentration of fiber. J. Dairy Sci., 72, 2301-2307.

MALESTEIN A., VAN'T KLOOSTER Th.A., PRINS R.A. CONOTTE G.M., 1984. Concentrate feeding and ruminal fermentation - 3 - influence of concentrate ingredients on $\mathrm{pH}$, on DL lactic concentration in the rumen fluid of dairy cows and on dry matter intake. Neth. I. Agric. Sci $32,9-21$.

MARTZ F.A., BELYEA R.L., 1986. Role of particle size and forage quality in digestion and passage by cattle and sheep, J. Dairy Sci., 69, 1996-2008.
NAJAR T., GIGER S., PONCET C., SAUVANT D., 1988. Variations du temps de séjour du foin, du son de blé et de la pulpe de betteraves avec le niveau alimentaire et la proportion d'aliment concentré dans une ration mixte chez des chèvres laitières. Reprod. Nutr. Develop., 28, 113-114.

NORGAARD P., 1981. Foderstrukturens indflydesle pa laktevende koers tyggeaktivited og vommotorik, Thesis, Roy. Vet. Agric. Univ., Copenhagen

NORGAARD P., 1983. Optimale Foderrationel til malkekoen, Fodervaerdi, Foderoptagelse, omsaetning og produktion, Rep. 551 Nat. Inst. Anim. Sci. Copenhagen.

PLATKOWSKI B., NAGEL S., BERGNER E., 1977. Das wiederkauverhalten von kuben bei unterschiedlichen Trockensubstang aufnahme und verschiedener physikilischer, Arch. Tierernähr, 27, 563-569.

PONCET C., GOMEZ L, MICHALET-DOREAU B., GEAY Y. 1987. Temps de séjour comparé du foin du son de blé et de la pulpe de betterave, d'une ration mixte distribuée à deux niveaux d'alimentation à des moutons et des taurillons, Reprod. Nutr. Develop., 27, 219-220.

REMOND B., 1978. Tableau p. 181 in «L'Alimentation des ruminants ». Ed. INRA Publications - Versailles 78000 .

SAKURAI K., FUIISHIRO S., SHINIOH K., HONZAWA N., YAMAMOTO F., TOMABECHI T., YOSHIDA $M$. TAKAHASHI A., ITABASHI A., ABE A., 1989. Effect of chewing time of diets on rumen fermentation and milk production in dairy cows. A.J.A.S., 2, 416-417.

SHAVER R.D., NYTES A.J., SATTER L.D., JORGENSEN N.A. 1986. Influence of amount of feed intake and forage physical form on digestion and passage of prebloom alfalfa hay in dairy cows, J. Dairy Sci., 69, 1545-1559.

SUDWEEKS E.M., ANDERSON M.J., LAMB R.C., LAW S.E., ELY L.O., Mc CULLOUGH M.E., SISK L.R., 1979. Development and application of a roughage value index for formulating dairy rations. Georgia. Exp. Sta. Res. Bull 238.

SUDWEEKS E.M., ELY L.O., MERTENS D.R., SISK L.R., 1981. Assessing minimum amounts and form of roughages in ruminants diets: roughage value index system. I. anim. Sci., 53, 1406-1411.

TELLER E., VANBELLE M., 1989. Voluntary intake of grass silage in relation to chewing activity and rate of disappearance from rumen in dairy breifers. A.J.A.S., 2, 307-308.

TELLER, E., VAN EYS J., VANBELLE M., 1990. Effect of $\mathrm{NaOH}$-treatment and of mean particle size on ruminating index of wheat straw in all concentrate diet for finishing cattle. Reprod. Nutr. Develop. (sous presse).

ULYATT M.J., DELLOW D.W., JOHN A., REID CSW, WAGHORN G.C., 1984. Contribution of chewing during eating and rumination to the clearance of digesta from the rumino reticulum. In "Control of digestion and metabolism in ruminants". Ed. Milligan, Grovum, Dobson, Prentice-Hall, p 498-515.

VAN SOEST P.J., FOX D.G., MERTENS D.R., SNIFFEN C.J., 1984. Discounts for net energy and protein. Fourth revision Proc. Comell Nutriton Conf., Syracuse, N.Y. p. 121-136.

WOODFORD J.A., JORGENSEN N.A., BARRINGTON G.P., 1986. Impact of dietary fiber and physical form on performance of lactating dairy cows. J. Dairy Sci., 69, 1035-1047

WOODFORD S.T., MURPHY M.R., 1988. Dietary alteration of particle breakdown and passage from the rumen in lactating dairy cattle. J. Dairy Sci., 71, 687-696.

\section{Summary}

The fibrosity index of ruminant feed and diet.

The fibrosity index (FI) of feed and diet is equal to the chewing time (intake and rumination) expressed per $\mathrm{kg}$ of dry matter $(\mathrm{mn} / \mathrm{kg}$ DM). A decrease of the FI of a diet is associated with a lower ruminal motricity and salivary secretion, it is moreover related to a decreased milk fat content and an increase in the risk of digestive disorders. There is a large range of FI values for feeds (0 to $250 \mathrm{mn} / \mathrm{kg}$ DM). According to published information the FI concept can only be used as an indication, not as a precise feed unit.

SAUVANT D., DULPHY J.P., MICHALET-DOREAU Brigitte. 1990. Le concept d'indice de fibrosité des aliments des ruminants. INRA Prod. Anim., 3 (5) $309-318$. 